\title{
On the Organisation of Geographical Knowledge: data models for gazetteers and historical GIS
}

\author{
Humphrey Southall, Alexander von Lünen, Paula Aucott \\ Department of Geography \\ University of Portsmouth, UK \\ \{Humphrey.Southall,Alexander.von-Luenen,Paula.Aucott\}@port.ac.uk
}

\begin{abstract}
Historical documents contain many geographical names, and text describing geographical relationships, but few coordinates or accurate maps. Historical GISs consequently contain much conjecture and anachronistic data. The paper outlines an alternative approach based on a formal representation of the textual information.
\end{abstract}

\section{Introduction}

Almost by definition, "geographical information" is coordinate data; but also by definition, history as distinct from archaeology is about documentary evidence. Most historical documents contain no maps, and historical maps are often an unreliable source of coordinates. Instead, the "geographical knowledge" embedded in historical documents consists mainly of geographical names and textual statements of spatial relationships. Conventional historical GIS seeks to rapidly convert this information into coordinates, which often leads to questionable assignments of places to locations and a spurious sense of accuracy.

The knowledge organisation systems literature provides a taxonomy of taxonomies. The simplest gazetteer is just a "word list", lacking hierarchies or other relationships between entities. More complex gazetteers can be thesauri or ontologies, and this paper presents two linked examples: an ontology of European administrative units, from states to parishes, and a more tentative ontology of British "places". Both can include coordinates but do not require them, so covering "places" with unknown locations.

Both these structures form part of the Great Britain Historical GIS (GBH GIS), which aims to provide a systematic spatial framework for historical information about Great Britain. It is derived from sources which cover all or most of Great Britain, although it has expanded to include experimental information on other European countries. Despite beginning life as a fairly conventional Geographical Information System (GIS) it has evolved into an ontology-based structure for identifying and storing information about geographical entities. This framework can be utilised behind different interfaces for a variety of purposes, such as the 'A Vision of Britain through Time' website which presents compiled geographical information on individual localities in Great Britain or the Timespatial Query and Visualisation Interface (Qviz; www.qviz.eu) developed as a prototype European cultural heritage portal. This article explores the motivation and methodology employed in this innovative approach to a historical GIS with no polygons.

\section{Data Models for Historical GIS}

Mainstream GIS is based around a data model consisting of arcs, points or polygons. Attribute data must have a relationship with the spatial data, and cannot exist except where a spatial data item exists. There can be any number of spatial items and each one can have multiple pieces of attribute data. Any number of spatial items can be combined to create a "coverage" and all items must have the same geographically-locating information to be viewable simultaneously in the same coverage.

There has been considerable dissatisfaction with the data model described above. Firstly, this model is highly proprietary, as it is closely tied to the software products of one company, ESRI. Whilst this company has opened up the data format side of their GIS applications, allowing other software to interface with their GIS suite, the relationship between polygons and datasets imposed by the Shapefile format have limited the range of applications. Alternatives were therefore sought to allow for the expression of more diverse 
data, for example geographical entities not necessarily denoted by geometric abstractions.

In 2000, the Open Geospatial Consortium (OGC) introduced the Geographic Markup Language (GML) to define "an XML encoding for the transport and storage of geographic information, including both the geometry and properties of geographic features" [1], [2]. This standard has since matured into a widely accepted format within the GIS community.

In recent years, the "Semantic Web" has been increasingly discussed and adopted. Enriching Semantic Web Services with spatial information has also been widely sought by data providers. For example the "Semantic Web Interest Group" (SWIG) of the W3C have started work on a "Basic Geo Vocabulary" [3], [4]; a basic Resource Discovery Framework (RDF) vocabulary to provide a namespace for representing geographic coordinates.

The British Ordnance Survey (OS) has also started to define a set of ontologies for describing various spatial relationships, the Ordnance Survey Ontologies [5]. These are extensive, ranging from Administrative Geography to Topography and Hydrology. However, none provide a temporal, let alone a historical, dimension. This dimension is essential for creating an ontology for historical administrative units, as has been implemented in the GBHGIS.

\section{Ontologies in Historical GIS}

The already mentioned shortcomings of the Shapefile data model become all too apparent when applied to historical GIS (HGIS). The polygon-centric data model found in standard GIS packages is already poorly suited for temporal GIS. A HGIS, however, is distinct from a temporal GIS. HGIS require different nomenclatures for handling temporal data, as historical sources are often uncertain and imprecise. There could also be competing and contradictory historical data for the same locality. A HGIS should therefore allow for such contested datasets, although they would appear as redundant to most non-historians. Since historical analyses and interpretations are hard to achieve in a HGIS (or any other computer based system), they should focus on storing the "raw" data, i.e. competing datasets must be included rather than a single interpretation.

A standard GIS is inadequate for historical data as the latter often has little or no accurate coordinate data attached. Descriptions of localities in historical sources are usually based on common sense, i.e. are referred to by linguistic conventions rather than geographic correctness or geometric datasets. The mention of
"Stratford on Avon" in a historical text might refer to a certain part of the town of Stratford (i.e. the place "Stratford" in a wider sense), but it could also refer to a specific administrative unit named after "Stratford". Defined boundaries may be available for that unit, but the further we go back in time the less likely they are to be available. In any case, a historic narrator would have unlikely used such geographic descriptions, and rather referred to places as they were earmarked in contemporary culture.

Recently, ontologies have been much discussed by the HGIS community. Kauppinen et al [6], for example, devised an ontology-based historical GIS for Finland. While offering an interesting approach to HGIS, systems like this provide no answer to key questions encountered during the creation of a historical ontology. The most important is how to handle uncertainty within the data, the key difference between a specifically historical GIS and a merely temporal GIS. A historical GIS must be capable of coping with uncertain or even contested knowledge, especially at the level of international entities. European countries with their diverse and complex history of administration offer many contentious or ambiguous cases, particularly when it comes to countries with a history of foreign occupation. While countries like Sweden have straightforward administrative histories, Estonia came under the authority of three administrations of varying durations in the mid to late 20th century alone: as part of Germany, then of the USSR and finally as an independent nation. A system dealing with the administrative history of Estonia must therefore be able to accommodate historical changes in administrative relationships. Fortunately, although Great Britain has not had a history of foreign occupation, other aspects of the history of British administrative geographies are very complex, and the GBH GIS already contained several mechanisms for handling historical complexity. Even so, the case of Estonia stretched the system to its limits. Before discussing these complexities, we will outline the general architecture of the Administrative Unit Ontology as developed by the GBH GIS.

\section{Rebuilding the GBH GIS as an ontology}

The original GBH GIS was a fairly conventional polygon-based GIS, innovative primarily through its inclusion of a full time dimension [7]. It was redesigned in 2002-3 as an ontology-based digital gazetteer, but at that time there was no formal standard for such a system. 
The Dublin Core Metadata Initiative (name space dcterms) [8] define some rudimentary spatial relationships, e.g. dcterms:isPartOf or dcterms:spatial. While Dublin Core is geared towards cultural heritage collections, these predicates can be used in different contexts, and Kauppinen et al take this approach by importing the Dublin Core into their ontology.

The Dublin Core ontologies were among the first to be devised to describe resources in libraries, etc. Another similar ontology is the CIDOC "Conceptual Reference Model" (CRM) [9], designed to account for "heterogenous cultural heritage information". Its primary usage is in museums, and the CRM provides an ontology to record museum and archive items, including their entry into the relevant collection. Starting as a relational data model in 1995, the CRM was re-designed as an ontology after 2000, and has become an ISO standard [10]. CIDOC-CRM is far more extensive than Dublin Core, and could therefore serve as a potential basis for future HGIS ontologies. The CRM consists of some 80 classes and 130 properties, which describe a wide range of entities encountered when dealing with historic information.

The GBHGIS was originally created in 1994 based on a standard ArcInfo database. By 2001, the limitations of this system had become apparent and the creation of a new, improved system began. The data in the old system were migrated when Semantic Web technology and corresponding ontologies were still in their infancy. The new system could base itself on comparably few paradigms and had to be designed with a number of compromises on efficiency and software due to availability.

Since our projected system differed significantly from other designs, we concluded that existing ontologies were inappropriate, so we created an ontology that was better suited for the domain. Data modelling and storage was consequently done in a relational database, with entity relationship modelling (ERM) in place. Luckily, ERM and ontologies share a common ground. Both are based on formal logics and a subset thereof, so-called "description logics" (DL). As Borgida [11] has shown, any ERM can be transformed into a DL language, from which the creation of an ontology is straight-forward, as ontologies are one application of DL. The fact that the ERM version of the AUO preceded the actual formal ontology therefore imposed no hindrance to the creation of the latter. The AUO can be extracted from the database with a custom Java applet, utilizing the Jena Semantic Web Framework [12].
Both the GBHGIS systems are stored in a RDBMS to support legacy applications that must run against the database, and for efficiency. This poses no trouble in practice because of the aforementioned interchangeability of ERM and ontologies.

\section{Administrative Unit Ontology Structure}

The Administrative Unit Ontology (AUO) is the framework upon which the rest of the system is constructed. Each element of an administrative geographical entity (unit) is broken down into components which are linked together via a numerical identifier. These elements are:

- Existence and type

- Names

- Relationships to higher and lower level units

- Boundary polygons

- Statuses (detailed type)

Each of these records includes dates and sources. We also identify "jurisdiction", i.e.who is entitled to edit each record.

At the heart of this framework each unit must have at least one preferred name, one IsPartOf relationship, a single unit type and an authority for its legal existence. Beyond that any number of other combinations of data content may or may not exist.

We define the following relationship types:

- AbolishedToCreate: one unit was discontinued to form part of a new unit;

- AbolishedToEnlarge: area of a discontinued unit was merged with another pre-existing unit;

- AdministeredBy: in 20th century Britain, a Local Government District could have shared administrations;

- SucceededBy: a relationship to the unit's successor;

- ReducedToCreate: a unit's boundaries was reduced to create a new unit;

- ReducedToEnlarge: one unit's area was reduced to enlarge that of another unit.

- BoundaryChange: non-specific boundary change, if not covered by the above;

- Event: any event affecting a unit other than boundary change;

The great majority of relationships were of type IsPartOf, but the other relationship types proved useful for describing some of the more complex relationships. Each unit and relationship comes with a duration, dummy values of "9999" being used to denote the unit is still in existence, and " 0 "where the date of creation is unknown. 


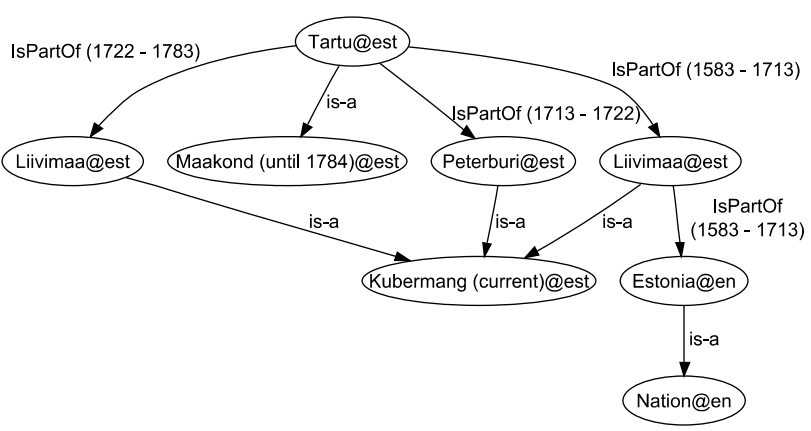

Fig. 1 Second level relationships for the historic Estonian county of "Pärnu".

The figure shows the relationships of the specific historic Maakond of Pärnu as it existed until 1784. This county was part of the duchy/guberniya (Estonian: Kubermang) of Livonia (Estonian: Liivimaa) from 1583 to 1713 . During the "Great Northern War" (1700-1721) From 1713 to 1722 it was part of the Swedish province of Livonia (Estonian: Liivimaa), but the Russian Empire seized control of the province when it pushed the Swedes back during the course of the war. With the "Treaty of Nystad" at the end of the war, Livonia was restored as a guberniya, but remained under Russian control [13]. This rather complicated relationship to other units explains the unit type "Nation" in the AUO. We use this type to describe a unit at the national level that did not exist as an independent state. Estonia was not an autonomous state for long stretches of its history, being occupied by Denmark (partly), Germany (twice), Sweden, the Russian Empire, and the USSR, with independence only between 1918 and 1940 and from 1991 onwards. We therefore define two separate "States" called Estonia covering those periods of independence, but also define a "Nation" called Estonia with a continuous existence to which we have attached all other Estonian relationships.

\section{Towards a Place Ontology}

An ontology of administrative units meets the primary needs of the archive sector for geographical information, as most archives exist primarily to preserve the records of government bodies, and in Britain the commonest kind of archives are county and other local record offices, funded by territorial administrative units. The records of most interest to family historians were mostly created by and indexed via administrative units, especially parishes and, in England, Registration Districts.

Administrative units are also very relevant to cataloguing one particular kind of library content, official statistics, and the GBH GIS originated as a framework for historical census and vital registration statistics. However, most geographical names appearing in books are not references to legally defined administrative areas, to States and Counties and Districts, but to much less formal regions, localities and neighbourhoods. These pose additional challenges.

An ontology of administrative units has some value as a more general gazetteer of places: towns and cities of any size have almost always given their names to an administrative unit at some point in their history, in England the same is true of most villages and many hamlets, and the names of current English local authorities identify some broader regions as well: "New Forest" or "High Peak". However, we have only to move to Scotland to find many large villages and some towns which never feature in lists of administrative units.

The larger problem, however, is that almost all towns and many villages gave their names to whole series of administrative units. The Vision of Britain web site includes a large library of historical British travel writing, but when Daniel Defoe, for example, visited Reading in Berkshire he did not visit the Hundred of Reading, the Urban Sanitary District of Reading or any other of the thirty units we currently know as named after it, he visited the place called Reading.

Our immediate solution was to construct a simplified "places gazetteer" from the AUO, by writing software which grouped administrative units together based on similarity of name and location. This worked well for Reading but proved more problematic elsewhere. For example, this software never did work out that "Hull" and "Kingston upon Hull" was one and the same.

We are now constructing an independent gazetteer of places, beginning by computerising an existing published gazetteer from the 1950s but linking it to the existing set of places derived from the AUO. This will be a true ontology, with a simple typology of relationships, "Within" and "Near", and holding many different names for the same place. This is unquestionably more challenging than building an ontology of administrative units defined, named, bounded and assigned to types by legal processes.

In our first version of this system, the hierarchy of "Within" is based on defining counties then states as higher-level "places", and locating low level "places" within them via point-in-polygon queries. Our "near" hierarchy is a little more interesting: we began by assigning a population to as many places as possible, then developed an algorithm which began by finding 
ten top-level places with large populations but dispersed around the country, then for each of these ten identifies a set of ten second-level places nearby but smaller, and so on until all places were assigned.

\section{Conclusion}

This paper describes the construction of a large and complex reference resource, an Administrative Unit Ontology designed to record the detailed administrative geography of the whole of Europe, and already documenting Great Britain, Sweden and Estonia in great detail. The AUO currently contains 78,471 entities, 126,121 geographical names and 249,241 relationships, and there seem to be no technical reasons why it cannot scale further. It is better suited than a conventional GIS to the special needs of historians and the cultural heritage sector: while it can include detailed information on administrative boundaries it does not require them, and a unit can be included based on a single appearance in, for example, a medieval taxation list; we can hold dates of greatly varying degrees of precision; we can hold any number of names for each unit, recording their language and identifying preferred and alternate forms. It does however require more careful selection of entities for inclusion than does a conventional GIS.

We noted that building web sites around formal ontologies rather than GIS frameworks led to much better findability in search engines, and consequently much higher usage.

Geographical ontologies are relevant, but many historians are interested in "places", not administrative units. While administrative units are defined in law, and tend to be well documented in official records, "places" are defined ultimately in consciousness and discourse, making the construction of "place ontologies" more challenging, especially if they are to include places and place names which existed only in the past, not today.

\section{References}

[1] OGC (2000), 'Geography Markup Language (GML) v1.0', OGC Document Number: 00-029, Open Geospatial Consortium, http://portal.opengeospatial.org/files/?artifact_id=7197, accessed Nov 2008.

[2] Badard, T. \& Richard, D. (2001), 'Using XML for the exchange of updating information between geographical information systems', Computers, Environment and Urban Systems 25, 17-31.

[3] W3C (2003): 'Basic Geo (WGS84 lat/long) Vocabulary [Website]', W3C Semantic Web Interest Group. http://www.w3.org/2003/01/geo/, accessed Nov 2008.

[4] Kresse, W. \& Fadaie, K. (2004), ISO Standards for Geographic Information, Springer, Berlin.

[5] OS (2009), 'Ordnance Survey Ontologies [Website]', Ordnance Survey, UK, http://www.ordnancesurvey.co.uk/oswebsite/ontology/, accessed Feb, 2009.

[6] Kauppinen, T.; Väätäinen, J. \& Hyvönen, E. (2008), Creating and Using Geospatial Ontology Time Series in a Semantic Cultural Heritage Portal, in 'S. Bechhofer et al.(Eds.): Proceedings of the 5th European Semantic Web Conference 2008 ESWC 2008, LNCS 5021, Tenerife, Spain', pp. 110-123.

[7] Gregory, I. \& Southall, H. (1998), 'Putting the Past in Its Place: the Great Britain Historical GIS', in S. Carver (ed.), Innovations in GIS 5 (London: Taylor \& Francis).

[8] DCMI Usage Board (2008), 'DCMI Metadata Terms', Dublin Core Metadata Initiative, http://dublincore.org/documents/dcmi-terms/, accessed Feb 2009.

[9] http://cidoc.mediahost.org/home(en)(E1).xml, accessed 09 March 2009.

\section{[10] ISO21127:2006.}

[11] Borgida, A.; Lenzerini, M. \& Rosati, R. (2003), Description Logics for Databases, in Franz Baader; Diego Calvanese; Deborah McGuiness; Danielle Nardi \& Peter Patel-Schneider, ed.,Description Logic Handbook : Theory, Implementation and Applications, Cambridge University Press, Cambridge.

[12] http://jena.sourceforge.net. 